\title{
Rare FCNC-B Decays at Hadron Machines*
}

\author{
David B. Cline \\ University of California Los Angeles, Center for Advanced Accelerators \\ Physics Department, 405 Hilgard Avenue, Los Angeles, CA 90024-1547
}

September 13, 1993

UCLA-CAA-00104-8/93

\begin{abstract}
We review the current studies and future prospects for studies of Flavor Changing Neutral Current (FCNC) processes, emphasizing rare $B$ decays. These experiments will be very sensitive to physics beyond the standard model. A key problem in these studies is the method to trigger the detector. We discuss a novel technique that uses an Optical-Cherenkov light trigger to select events with significant impact.
\end{abstract}

\section{Contents}

1 Introduction 1

2 Overview of the FCNC Amplitude Limits 1

3 New Rare $B$ Studies 3

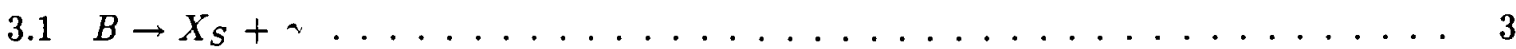

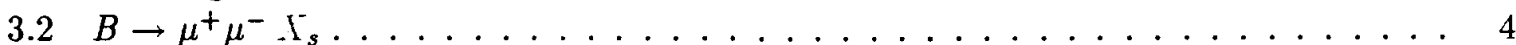

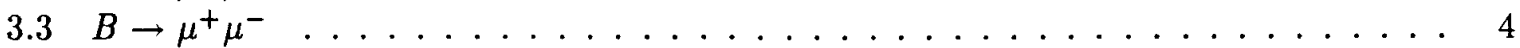

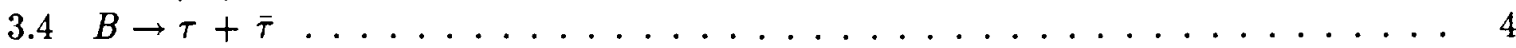

4 Sensitivity of the FCNC to New Physics 5

5 Novel Triggers for Beauty Event Selection 5

6 Summary 6

7 Acknowledgement 6

\section{Introduction}

The early search for FCNC with $K$ decays helped motivate the standard model and the GIM mechanism $[1,2]$. The studies are continuing. For example, we show in Fig. 1 the ongoing search for

$$
K^{+} \rightarrow \pi^{+} \nu \bar{\nu}
$$

\footnotetext{
•Invited Review Article for Modern Physics Letters A.
} 
that has been in progress for 30 or so years $[3,4]$. However, it now appears that progress will be made in learning more about the standard model by studying FCNC processes with much heavier quarks, such as charm or beauty. In this article I will restrict the discussion to $B$ decays, however, charm FCNC processes are also of great importance. Many theories that go beyond the standard model predict much larger FCNC effects in the heavy quark system or perhaps for processes that involve the 3 rd family entirely.

\section{Overview of the FCNC Amplitude Limits}

Current branching ratios for FCNC limits are order of magnitude $\sim 10^{-9}$ for $\mathrm{K}$ decays and order of magnitude $\sim 10^{-5}$ for $\mathrm{B}$ decays (see Table 1 ). These are also the levels expected for higher order corrections to the standard model. Thus the search for forbidden processes that indicate FCNC is nearly exhausted.

We can parameterize these results conveniently by considering that the weak neutral current processes are described by a flavor changing matrix, as in the case for the charged current reactions. In the case of FCNC however, we have up-quark transitions (i.e., $u \rightarrow$ charm) and down-quark transitions (i.e., $b \rightarrow s$ or $b \rightarrow d$ ). We show this simple parameterization in Figure 2a by analogy with the CKM matrix (Figure 2b). In this report we discuss methods to search for the off diagonal elements of the $D_{p g}$ and $U_{p g}$ matrices. We note that there have been other analyses of these off diagonal elements but we adopt the form of Fig. 2a for uniformity. We can put limits on the various off diagonal elements in the matrix using the following processes[5]

$$
\begin{aligned}
& K_{L}^{\circ} \rightarrow \mu^{+} \mu^{-}: \\
& \frac{B r\left(K_{L} \rightarrow \mu \mu\right)_{\mathrm{FCWNC}}}{B r\left(K^{+} \rightarrow \mu \nu\right)}=2\left[\frac{\tau\left(K_{L}\right)}{\tau\left(K^{+}\right)}\right]\left[\left(\frac{1}{2}-\sin ^{2} \theta_{w}\right)^{2}+\left(\sin ^{2} \theta_{w}\right)^{2}\right] \\
& \frac{\left(\operatorname{Re} D_{d} s\right)^{2}}{[V u s]^{2}}<2 \times 10^{-9} \\
& :\left|\operatorname{Re} D_{d s}\right| \leq 2.5 \times 10^{-5} \\
& B_{d} \rightarrow \mu^{+} \mu^{-} X: \\
& \frac{B r(B \rightarrow \ell \bar{\ell} X)}{B r(B \rightarrow \ell \nu x)} \quad\left[\left(\frac{1}{2}-\sin ^{2} \theta_{w}\right)^{2}+\left(\sin ^{2} \theta_{w}\right)^{2}\right] \\
& {\left[\frac{\left|D_{b d}\right|^{2}+\left|D_{b s}\right|^{2}}{\frac{1}{2}\left|V_{c b}\right|^{2}}\right]<6 \times 10^{-5}(90 \% \text { C.L. })} \\
& :\left|D_{b d}\right|<1.5 \times 10^{-3} \\
& \frac{B r\left(D \rightarrow \mu^{+} \mu^{-} X\right)}{B r\left(D \rightarrow \mu \nu X_{s}\right)}=\left[\left(\frac{1}{2}-\sin ^{2} \theta_{w}\right)^{2}+\left(\sin ^{2} \theta_{w}\right)^{2}\right]\left[\frac{\left|U_{c u}\right|}{\left|V_{c s}\right|^{2}}\right]<10^{-3}(4) \\
& :\left|V_{c u}\right|<1.6 \times 10^{-2}
\end{aligned}
$$

In the latter case a better limit is obtained from the limit on $\mathrm{D}^{\circ}-\overline{\mathrm{D}}^{\circ}$ mixing $\left(\left[\operatorname{Re} \mathrm{U}_{\mathrm{cu}}\right]<10^{-3}\right)$. These values represent the best present limits on the FCNC matrix elements. Limits on $U_{t c}, U_{t u}$ will have to wait for the discovery of the $t$ quark since the transitions $\mathrm{Z} \rightarrow t \overline{\mathrm{u}}$ or $\mathrm{Z} \rightarrow t \overline{\mathrm{c}}$ are nearly kinematically forbidden since we now have a limit of $\mathrm{M}_{t}>113 \mathrm{GeV}$. Thus the current limits on $\left|D_{d s}\right|:\left|D_{b s, b d}\right|:\left|U_{c u}\right|$ are order $2 \times 10^{-5}, 10^{-3}$ and $10^{-2}$ respectively. 
We illustrate the search for rare $B$ decays using $B \rightarrow \mu \mu x$ in the UA1 experiment. For the decay $B \rightarrow \mu^{+} \mu^{-} X$ and for the exclusive channel $B^{0} \rightarrow \mu^{+} \mu^{-} K^{0 *}$, the contributing diagrams are similar to those for $B^{0} \rightarrow \mu^{+} \mu^{-}$. However, the situation is slightly more complicated: for $B \rightarrow \mu^{+} \mu^{-} X$, both charged and neutral B mesons contribute; the resulting dimuons are part of three or more body decays, thus complicating calculations of the dimuon invariant mass $\left(M_{\mu \mu}\right)$ distribution; and there is an interference between the penguin-diagram decay and the resonance decay $B \rightarrow J / \psi$ or $\psi^{\prime}+X ; J / \psi$ or $\psi^{\prime} \rightarrow \mu^{+} \mu^{-}$. Figure 3 shows the calculated differential branching ratios $d B R\left(b \rightarrow \mu^{+} \mu^{-} s\right) / d M_{\mu \mu}$, for the combined decay (solid line) and for the penguin-diagram decays alone (dashed lines). The destructive interference above the $J / \psi$ and $\psi^{\prime}$ is clearly visible[5,6].

As can be seen in Figure 4, no evidence for an excess of events in our search region is observed. To determine our efficiency to this decay mode, we use the ISAJET program to simulate this process[6]. We force the $M_{\mu \mu}$ distribution of dimuons coming from the b-quark decay to be that of Reference 6 We find an overall efficiency of $(1.1 \pm 0.3) \%$ for dimuons coming from a parent $b$ or $\bar{b}$ quark of $P_{T}>6 \mathrm{GeV} / \mathrm{c}$ and $|y|<1.5$. Using the fitted curve of Figure $4 \mathrm{a}$ and $4 \mathrm{~b}$ we estimate the background to this decay mode to be $8.7 \pm 1.7$ events compared with 9 events seen. After folding in the systematic uncertainties and the uncertainty from the background subtraction, and using the UA1 b-quark cross-section, we obtain a $90 \%$ confidence level upper limit of:

$$
B R\left(B \rightarrow \mu^{+} \mu^{-} X\right)<5.0 \times 10^{-5}\left(B_{d}^{0}, B^{ \pm} \text {and } B_{s}^{0} \text { not separated }\right)[6]
$$

The search for $B \rightarrow \mu^{+} \mu^{-} X$ indicates the major problem facing the detection of rare $B$ decays, namely the large backgrounds that come from the generic process[7]

$$
\begin{aligned}
B \rightarrow & C+\ell+\nu_{e} \\
& \hookrightarrow \ell^{\prime}+\nu_{e^{\prime}}+S
\end{aligned}
$$

We will see that these processes will be the most difficult to eliminate for the process $B_{S}^{0} \rightarrow \tau+\bar{\tau}$ below $[\bar{\tau}]$

\section{New Rare $B$ Studies}

There are many theoretical models that give FCNC in Rare $B$ decays. The interesting $B$ decay branching ratios to be studied vary from $\sim 10^{-4}$ to $10^{-9}$. If we take the UA1 study reported above as an example, we would find overall detection efficiency no greater than $\sim 10^{-2}$. Thus, in order to detect these processes we need effective $B$ samples of $10^{6}-10^{11}$, at least. In order to study these processes we need at least $10^{2}$ events. Thus effective $B$ samples of $10^{8}-10^{13}$ are required. The only method by which this very large rate of $B$ production can be obtained is in very high energy hadron interactions, either at fixed target experiments or future colliders like the LHC or SSC. Extracting these rare $B$ signals requires methods to reduce the very large backgrounds and to overcome the severe trigger problem. We will return to these issues later in this report. We now turn to a brief discussion of the interesting rare $B$ decays that may be studied in the future.

\section{$3.1 B \rightarrow X_{S}+\gamma$}

A recent limit of $\sim 5 \times 10^{-5}$ has been placed on this decay by the CLEO II group[8]. A UCLA group has carried out a study of the detection of this decay at hadron machines[9]. Simulation studies are being conducted of the photons from the inclusive decay $b \rightarrow s \gamma$ using the Monte Carlo program PYTHIA to generate $B$ events. Since $b \rightarrow s \gamma$ decays are not implemented in PYTHIA, we treat the decay as a two body decay, assuming that the $s$ and spectator quarks hadronise into 
a state with a mean mass halfway between the $K^{*}$ and the $K_{4}^{*}$. This generates a mass distribution with FWHM equal to the $K_{4}^{*}-K^{*}$ mass difference. The shape is a Gaussian, truncated at the low end at the mass of the $K^{*}$ and at the high end at the mass of the $B$.

We assume an energy resolution of $(10 \%) / \sqrt{E}$, an angular resolution of $1 \mathrm{mrad}$ for $\gamma$ 's originating from the $b$ quark. The $b \rightarrow s \gamma$ photons were broadly peaked in the transverse momentum, with a mean of $2.3 \mathrm{GeV}$ and rms width of $0.8 \mathrm{GeV}$, while the photons from $B$ background events had a mean $p_{t}$ of $0.2 \mathrm{GeV}$, and the distribution fell off somewhat more slowly than exponential. The $p_{t}$ spectrum of $\gamma$ 's from $1 \mathrm{~K}$ non- $B$ events was also obtained to permit background subtraction. The transverse momentum of the photons had a mean $p_{t}$ of $0.17 \mathrm{GeV}$ and dropped off at a $p_{t}$ of $2.5 \mathrm{GeV}$.

The standard model prediction for the branching ratio of $b \rightarrow s \gamma$ is, according to Ali, $(2 \sim$ $5) \times 10^{-4}[10]$. Assuming a branching ratio of $4 \times 10^{-4}$, there should be 20 signal events corresponding to the $50 \mathrm{~K}$ background events we generated. Since even at the peak of the signal $p_{t}$ the background exceeds the signal by two orders of magnitude, we have investigated several cuts to see whether we can extract the signal from the background.

In order to suppress $\gamma$ 's from $\pi^{0}$, we exclude from the analysis all $\gamma$ 's which are consistent within the calorimeter resolution with combining with any other $\gamma$ in the event to form a $\pi^{0}$. Various $\gamma \gamma$ mass range cuts are examined. If the mass range $0 \leq m_{\gamma \gamma} \leq 0.2 \mathrm{GeV}$ is excluded, the signal to background ratio is improved by a factor of 142 , with a signal retention of $55 \%$.

After the $\pi^{0}$ mass range cut of $0 \leq m_{\gamma \gamma} \leq 0.2 \mathrm{GeV}$ is applied the background $\gamma$ 's from $\pi^{0}$ are entirely eliminated. This leaves high $p_{t} \gamma$ 's from $B$ or $D$ to $\eta$ decays, which may be over represented in PYTHLA, as evidenced by the apparent lack of such background in CLEO data.

Fig. 5 shows the $p_{t}$ distribution of $\gamma$ 's from $250 \mathrm{~K} B$ background events superimposed with $1 \mathrm{~K}$ $B$ signal events after the $\pi^{0}$ mass range cut of $0 \leq m_{\gamma \gamma} \leq 0.2 \mathrm{GeV}$ is applied. There are about 20 $\gamma$ 's at the high $p_{t}$ end, $\geq 3.5 \mathrm{GeV}$, and these are all from $\eta$ decays. Figure 5 shows the $p_{t}$ spectrum of $\gamma$ 's from $250 \mathrm{~K} B$ background events superimposed with $1 \mathrm{~K} B$ signal events, with the mass cut $0 \leq m_{\gamma \gamma} \leq 0.2 \mathrm{GeV}$ and with $\eta \rightarrow \gamma \gamma$ suppression. For the region $p_{t} \geq 3.6 \mathrm{GeV}$, the background is completely suppressed but the signal is statistically significant. The signal retention is $6.34 \%$.

Preliminary studies are also being conducted of the exclusive decay mode $B \rightarrow K^{*} \gamma$. First, all $K \pi$ combinations are formed and events in which the invariant mass is outside the range of the $K^{*}(0.7 \mathrm{GeV}$ to $1.1 \mathrm{GeV})$ are vetoed. Next we select $K \pi$ pairs consistent with the $K^{*}$ mass and form all combinations of these $K^{*}$ candidates and $\gamma$ 's which survived the $\pi^{0}$ mass cut. Then we apply the following cuts on the $K^{*}-\gamma$ : an invariant mass cut, a cut on the opening angle, and a primary vertex cut. Thus, it would seem that hadron machines can be used to detect $B \rightarrow x+\gamma$. The key issue is to provide a precision measurement of the branching fraction of this decay which is very sensitive to $m_{t}$ and many types of theories for beyond the standard model. For FNAL fixed target experiments, one can imagine detecting 100 - 500 events. For the LHC/SSC fixed target experiments the rate could be $10 \mathrm{~K}$ or more, allowing a precision measurement of this process.

\section{$3.2 B \rightarrow \mu^{+} \mu^{-} X_{s}$}

We follow the UA1 results which indicate that, in the mass range of $3.8-4.4 \mathrm{GeV}$, a signal may be detected[6]. For a branching ratio of $\sim 5 \times 10^{-6}$ we would expect that $\left(10^{10}\right) B \bar{B}$ production would be required to obtain a signal or $\sim 10$ events above background. Since the signal is background limited, it will require a careful study of the background and a detailed subtractor. This may be possible with the CDF or $D_{0}$ detectors at FNAL over the next few years. This would be very difficult for fixed target experiments at FNAL or u ven $e^{+} e^{-} B$ factories to detect. AT the LHC or SSC it should be rather straight forward. Some theoretical estimates for this process can be found in Ref. [6,10 and 11]. 


\section{$3.3 \quad B \rightarrow \mu^{+} \mu^{-}$}

This decay represents a process that is likely to provide an excellent signal, if the detector has adequate mass resolution, but will be severly rate limited if the branching fraction is $\sim 2 \times 10^{-9}$, as expected in the standard model.[10]. Again, using the detection efficiency obtained in the UA1 experiment for this decay mode of $\sim 4 \%$ and a branching fraction of $\sim 10^{-9}$ and a 10 events signal, we estimate that a sample of $10^{11} B \bar{B}$ events will be required to clearly detect this signal. Of course, models beyond the standard model could enhance this branching fraction by a factor of $\sim 10$, but not much more since we know that $B \rightarrow \mu^{+} \mu^{-} x^{-}$is within a factor of 10 of the standard model prediction[12]. Thus, an interesting search could be carried out when $\sim 10^{10} \bar{B} B$ events have been produced.

\section{4 $B \rightarrow \tau+\bar{\tau}$}

This process has only 3rd family particles and may be sensitive to new types of theories that are beyond the standard model. It also presents perhaps the greatest challenge for detection of any FCNC $B$ decay since the experimental signature is so complex and the branching ratio so small, even in beyond the standard model estimates. The estimated branching ratio in the standard model is $3.8 \times 10^{-7}[10]$. There are estimates for the branching ratio in some models that can go to $\sim 10^{-4}$. In addition, this process is not constrained directly by the UA1 limits on $B \rightarrow \mu^{+} \mu^{-} x$ and thus, any sensible search and limit is likely to be interesting. The method of detection and backgrounds for this process are discussed in Ref. [7]. We can summarize these results here. For the branching ratio $\sim 10^{-5}$, there seems to be little background, but at $\sim 10^{-6}$ the process

$$
\begin{aligned}
B_{s}^{0} \rightarrow & C_{s}+\tau+\bar{\nu}_{\tau} \\
& \hookrightarrow \bar{\tau}+\nu_{\tau}
\end{aligned}
$$

will likely become an important source of background.

In Ref. [7] some kinematic tricks are discussed to reduce this background. A Monte Carlo study similar to that for $B \rightarrow X+\gamma$ is being started at UCLA. Table 2 lists some of the possible rates for the various processes discussed here from the Snowmass workshop.

\section{Sensitivity of the FCNC to New Physics}

There are several classes of models that may have FCNC processes, as discussed in section 3 . We list them in a generic sense[7]

1. Supersymmetric theorem

2. Technicolor

3. Multiple scalars

4. 4th family

We believe that these four classes of theories covers most of the reports in the literature. However, it could be that the discovery of FCNC processes may be unpredicted by any extant theory and could lead to the correct theory.

There is a general belief that $F C N C$ must exist[13] in almost all models that go beyond the standard model, since the concept of Flavor Conservation[14] is ad hoc and the Higgs sector is more 
complex than that assumed by the standard model[14,15]. In Fig. 6 we show some of the diagrams that give the FCNC processes as discussed in section 3 from some of the theories, such as that in sections 3.1-3.4 above. Clearly, the search for FCNC in $B$ decays is of great importance.

\section{Novel Triggers for Beauty Event Selection}

The production of $\bar{B} B$ pairs at hadronic machines is very large. As a rule of thumb here are the kinds of rates expected per year for various machines:

a) Fixed Target at FNAL $\sim 10^{8} \bar{B} B /$ year

b) Colliding Beams at FNAL $\sim 10^{9}-10^{10} \bar{B} B /$ year

c) Dedicated Fixed Target Experiment at the LHC or SSC $\sim 10^{10} \bar{B} B /$ year

d) LHC or SSC Collider $\sim 10^{12}-10^{13} \bar{B} B /$ year

As a rule of thumb (from the UA1 experiment) the overall detection efficiency for rare processes is unlikely to exceed $\sim 10^{-2}$. Thus, in order to detect all the various decays discussed in section 3 , we will need the full range of $B$ production, up to the Super. Colliders.

The major issue in using these very large $\bar{B} B$ rates is the event trigger selection. The total hadronic event production per year will vary from $\mathrm{f} 10^{12}-10^{15}$. These events must be sorted through somehow to extract the $\left(10^{-5}-10^{-3}\right)$ fraction of $\bar{B} B$ events and then to find the rare events a factor of $10^{-4}$ to $10^{-9}$ is required. This is obviously an extremely difficult undertaking.

The key to the use of the large $\bar{B} B$ rates is the event trigger. We will discuss two concepts for event triggers that we believe hold promise for the FCNC studies and describe two extremes:

1. Trigger $J / \psi \rightarrow \mu^{+} \mu^{-}$events to tag a $\bar{B} B$ event[16]. This is currently being used in the E771 experiment at FNAL. On principle this could give a trigger with an efficiency of $\sim 10^{-3}$.

2. Trigger on the impact parameter displacement of charged tracks from the $B / \bar{B}$ decay using a fast Cherenkov light signal (optical trigger) $[17,18]$. Future use of a new type of photo detector (VLPC) may be important for this technique[19].

There are other concepts, like the detection of an impact parameter using a nearby silicon tracking array[20], and the expected different track multiplicity for events with $\bar{B} B$, and the total events, that we will not discuss.

Fig. 7a shows a schematic of the concept for the optical trigger. Basically, Cherenkov light from a displaced (impact parameter) track is trapped in the radiator and collected at the end with very sensitive photo detectors $[18,19]$. Fig. 7b shows some preliminary tests at CERN of this concept. The hope for this trigger is to give a factor of $10^{2}-10^{3}$ event selection, while maintaining a high detection efficiency for a broad class of $B$ decays. New tests are planned for the fall of 1993 at CERN.

\section{Summary}

We have illustrated in this brief review the importance of dedicated search for FCNC rare $B$ decays. We also showed that the small branching factors for these processes very likely require their study at hadron machines. In order for this to be accomplished, novel trigger techniques will be required. The optical trigger is on such clever idea. 


\section{Acknowledgement}

I wish to thank Jun Park and Jean Rhoades for their help with the simulations. I also wish to thank the members of the Rare $B$ decay group at the Snowmass workshop for interesting discussion.

\section{References}

[1] D. Cline, The Search for Weak Neutral Currents, Proc. of Ecol. Int. Hecg Novi (1967); Thesis at Univ. of Wisc. (1965).

[2] S.L. Glashow, J. Mliopoulos and L. Maiani, Phys. Rev. D2 1285 (1970).

[3] Proc. of Int. Symp. on 30 Years of Neutral Currents: from Weak Neutral Currents to the $(W) / Z$ and Beyond, to be publ. by AIP, editors D. Cline and A.K. Mann.

[4] J. Klems, et al., Phys. Rev. Lett. $\underline{24} 1086$ (1970).

[5] D. Cline, The Continuing Search for Flavor Changing Weak Neutral Currents, Cmnts. in Part. Phys. (1987); D. Cline, The Search for Rare $B$ Decays and $B-B^{0}$ Mixing, publ. in Proc. of 3rd Topical seminar on Heavy Flavors, San Miniato, Italy (1991); Proc. of the meeting on Rare $B$ Decays and Novel Flavor Factories, AIP book, July 1992 and the references therein; D. Silverman (private communication).

[6] C. Albajar, et al., Phys. Lett. B262 163 (1991).

[7] D. Cline, Report for the 1993 Snowmass Workshop on Rare $B$ Studies at Hadron Machines (to be publiched in proc.).

[8] Report from CLEO II group, Snowmass Workshop (1993).

[9] D. Cline, J. Park, J. Rhoads and M. Atac, Simulation Study of $B \rightarrow X \gamma$, UCLA preprint and to be published in proc. of the 1993 Snowmass Workshop.

[10] There are a large number of papers on $B \rightarrow X^{\prime}$ gamma and other Rare $B$ decays in the literature, we cite only A. Ali, G. Greub, and T. Mannel, Rare $B$ Decays in the Standard Model, DESY 93-016 (1993) and the references therein.

[11] N.G. Despande, et al., Phys. Lett. B308 322 (1993).

[12] For example see L. Randall and R. Sundrien, $b \rightarrow S \gamma$ and $B_{S} \rightarrow \mu^{+} \mu^{-}$in Extended Technicolor Models, MIT-CTP-2211 (1993).

[13] S. Pakvasa, Flavor Changing Neutral Currents: Then and Now, UH-511-770-93, and to be publ. in Proc. of the Symp. on 30 Years of Neutral Currents: from Weak Neutral Currents to the $(W) / Z$ and Beyond, AIP, edited by D. Cline and A.K. Mann.

[14] S. Glashow and S. Weinberg, Phys. Rev. D15 1968 (1977); E.A. Paschos, Phys. Rev. D15 1966 (1977).

[15] L. Hall and S. Weinber $\approx$, F'zvor Changing Scalar Interaction, UTTG-22-92; UCB 92/36.

[16] E771 Exper. at FNAL: see for example the report of R. Alexopoulous, et al., Nucl. Phys. B (Supp.) 27, 257 (1992). 
[17] G. Charpak, Y. Giomataris and L. Lederman, Nucl. Instr. Meth. A306 (1991); G. Charpak et al., Study of an optical trigger to be used for beauty search in fixed target mode at the LHC, CERN/DRDC/P30, 1991; G. Charpak et al., Addendum to the proposal DRDC/P30, Optical discriminator to be used for beautry search in fixed target mode at the LHC; experimental results and perspectives, CERN/DRDC/92-53, 1992; approved as RD30 on 12 Nov 1992.

[18] G. Charpah, et al., Experimental Study of an Impact Parameter Optical Discriminator, CERNPPE/93-94.

[19] M. Atac, et al., Nucl. Instr. Meth. A314 56 (1992).

[20] P. Schlein, et al., private communication. 


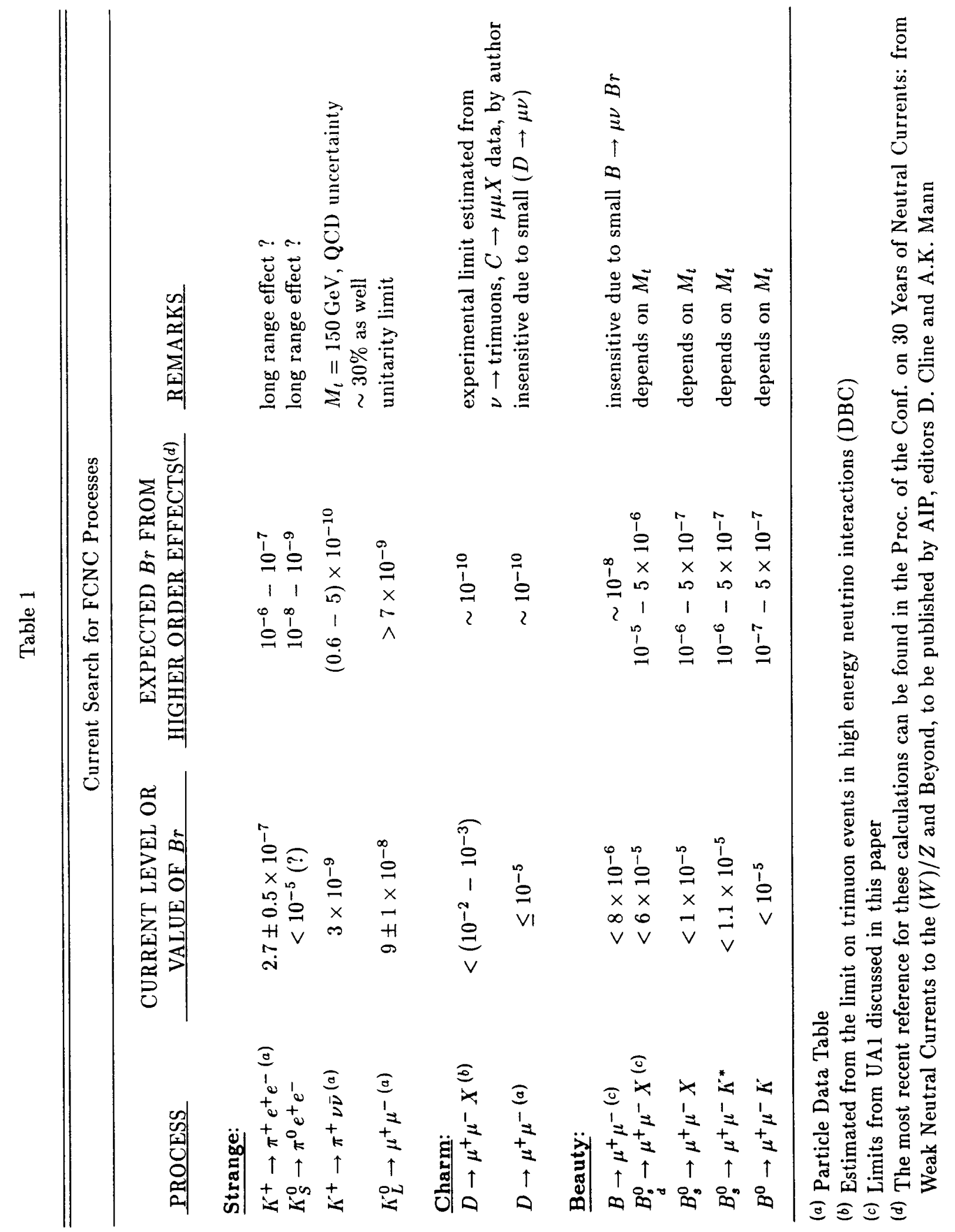




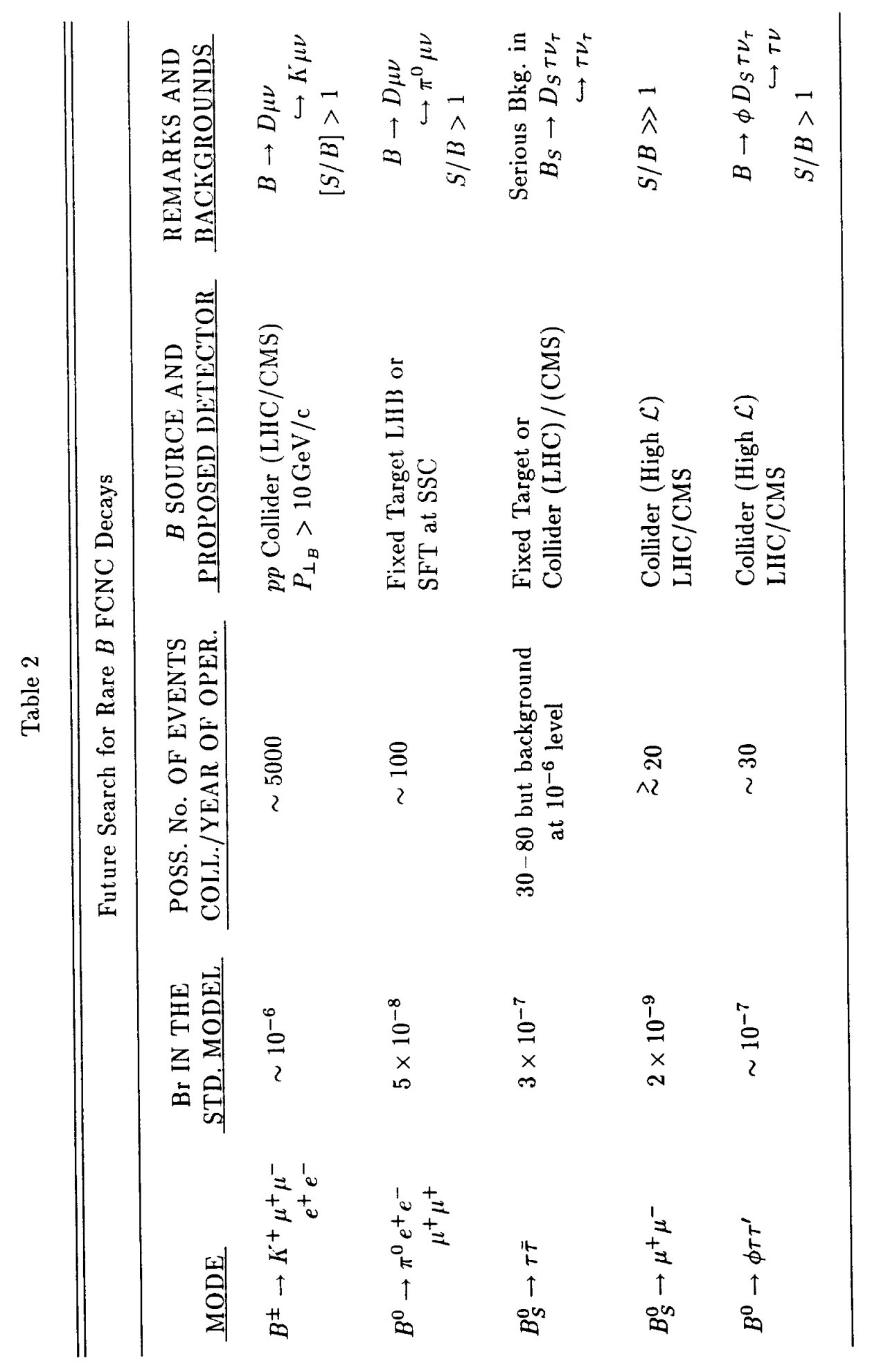


Table 3

Role of FCNC in the Rise of the Standard Model

PROCESS PERIOD IMPLICATION

\begin{tabular}{|c|c|c|}
\hline $\begin{array}{l}s \not t d \\
\left(K^{+} \rightarrow \pi^{+} \nu \bar{\nu}\right)\end{array}$ & $\sim 1963-1970$ & $\begin{array}{l}\text { FCNC absent at first level of Weak Interaction } \\
\text { [GIM mechanism] }\end{array}$ \\
\hline $\begin{array}{l}s \neq \text { Loop } f d \\
\left(K^{0}-\bar{K}^{0} \text { mixing }\right)\end{array}$ & $\sim 1960-1974$ & $\begin{array}{l}\text { New Quark in Loop, } m_{q} \sim 2 \mathrm{GeV} \text { are suggested } \\
\text { leads to invention of Charm quark }\end{array}$ \\
\hline$s+d$ & $\sim 1970$ 's & Some models for absence of FCNC imply that only \\
\hline$c+u$ & & $Q=1 / 3, Q=2 / 3$ quarks exist in nature \\
\hline$b+d$ & 1980's & Necessary existence of \\
\hline$b \nrightarrow s,\left(B \rightarrow \mu^{+} \mu^{-} X\right)$ & $\sim 1990$ 's & (massive) $t$ quark \\
\hline
\end{tabular}

$(u, d, c, s, b, t)$ all refer to quarks, and the process $s \neq d$ would indicate the current absences of a FCNC that would produce this transition. 


\section{HISTORY OF THE SEARCH FOR $\mathrm{K}^{+} \rightarrow \pi^{+} \nu_{\mathrm{e}} \bar{\nu}_{\mathrm{e}}-$ DIRECT}

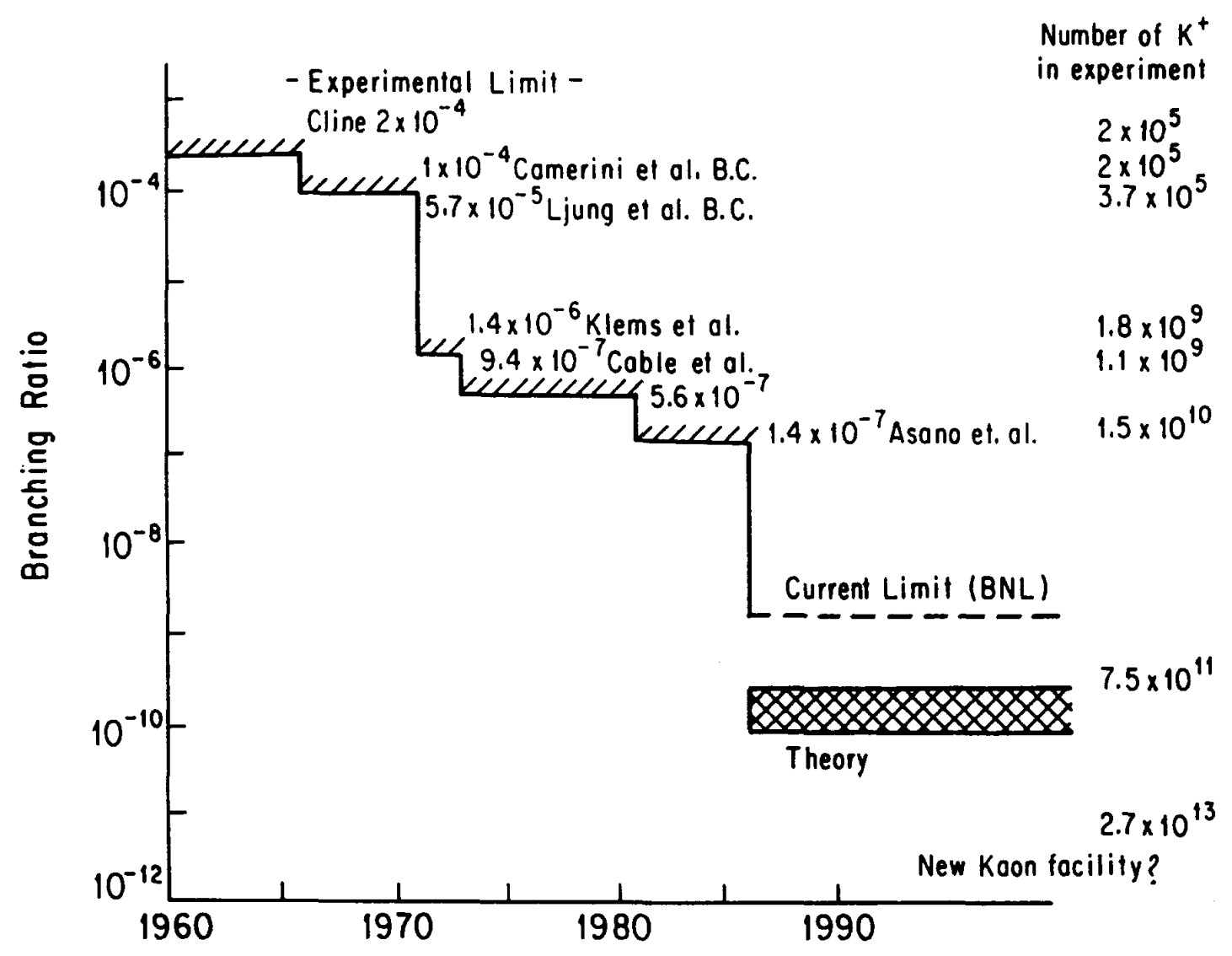

Fig. 1: History of the search for $K^{+} \rightarrow \pi^{+} \nu_{e} \bar{\nu}_{e}-$ direct 


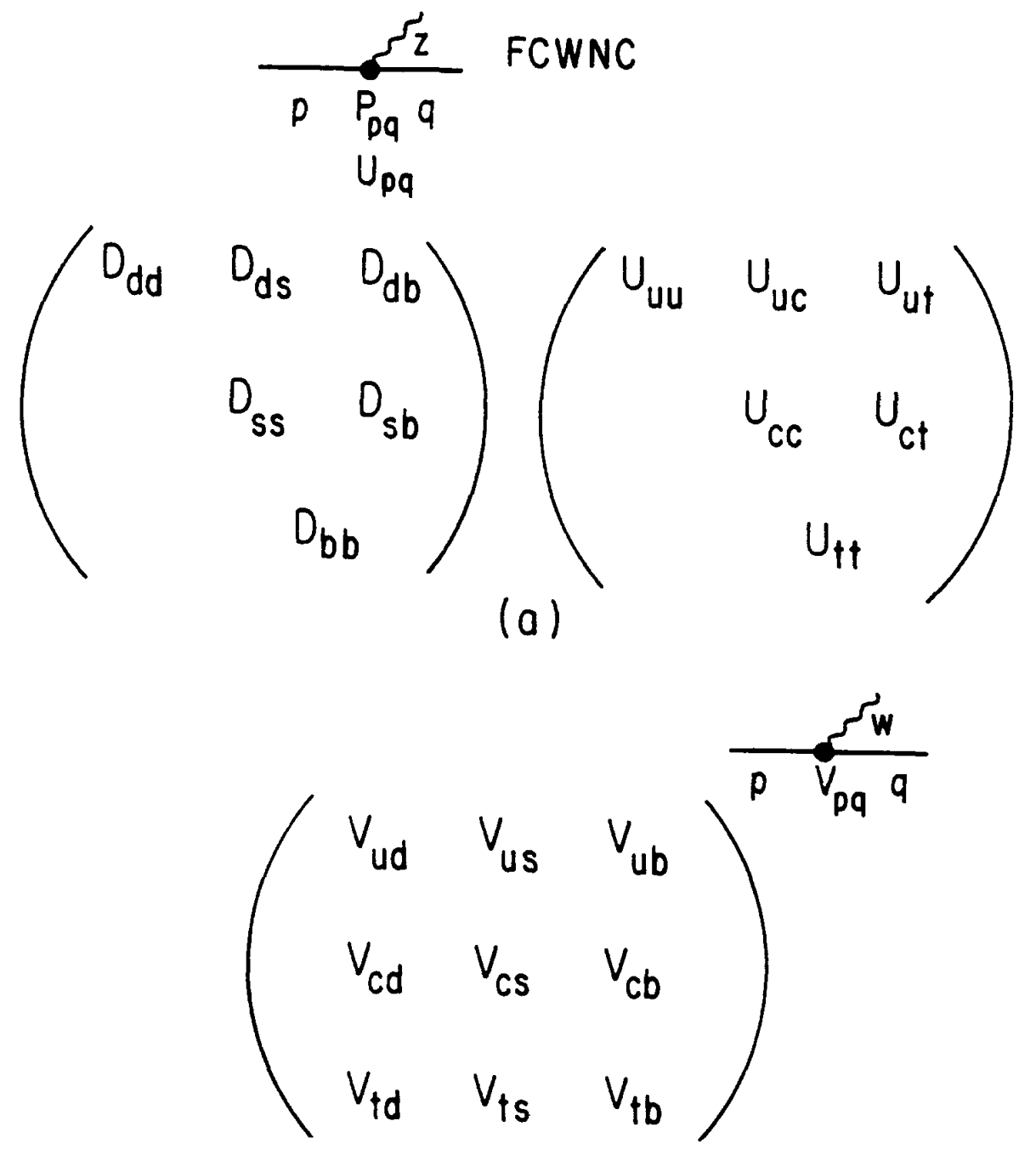

(b)

Fig. 2: (a) Suggested parameterization of FCNC using transition matrices for down quark $\left(D_{p g}\right)$ and up quark $\left(U_{p g}\right)$ elements. (b) Standard CKM matrix for charged current transitions. 


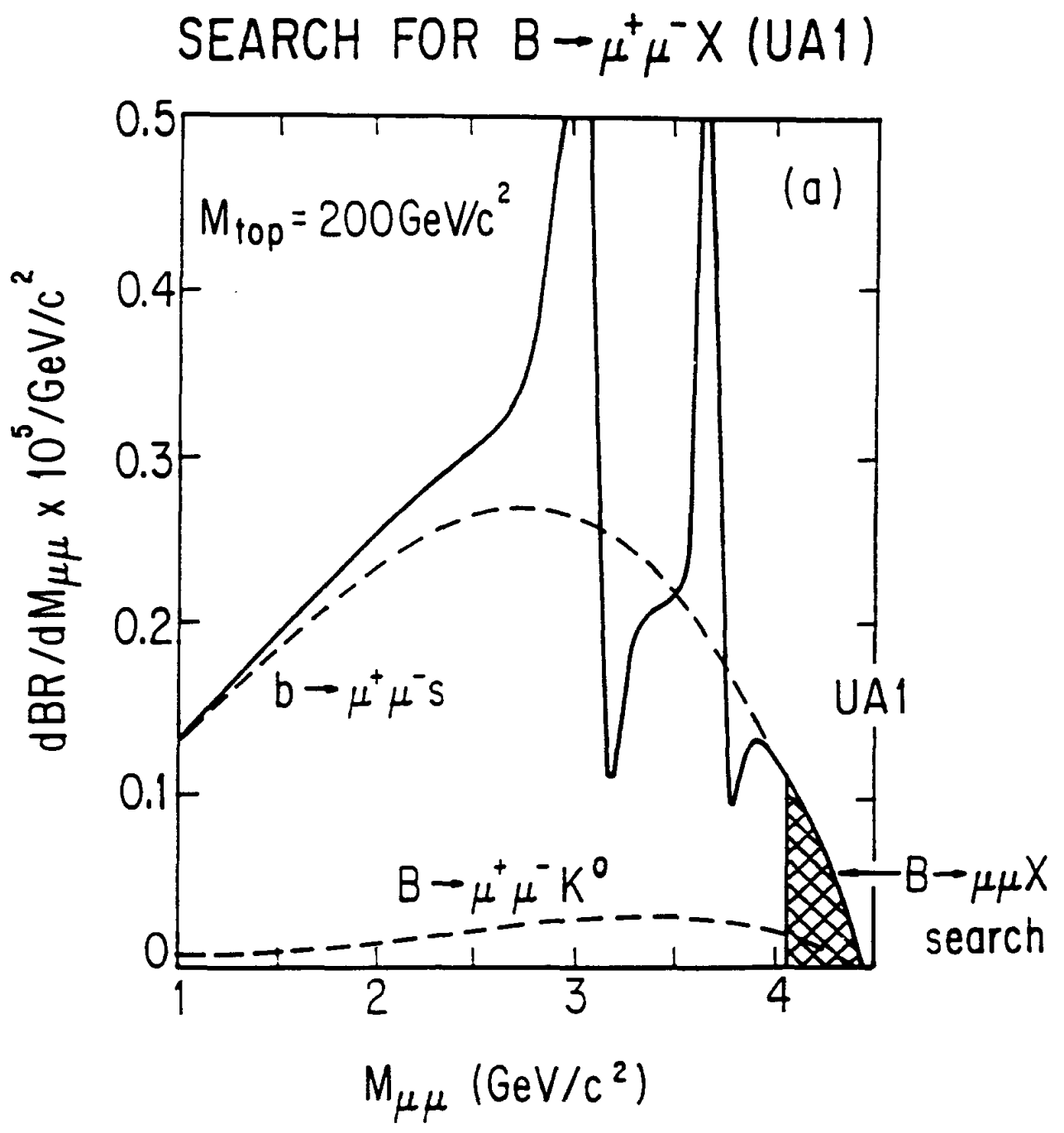

Fig. 3: The calculated differential branching ratio $d B r\left(b \rightarrow \mu^{+} \mu^{-} s\right) / d M_{\mu \mu}$ for a $200 \mathrm{GeV} / \mathrm{c}^{2}$ $t$-quark mass. The solid line corresponds to the differential branching ratio calculation for the combined resonance and penguin-diagram ${ }_{\triangleleft}$ decays; the dashed lines represent the penguin-diagram decays alone. 

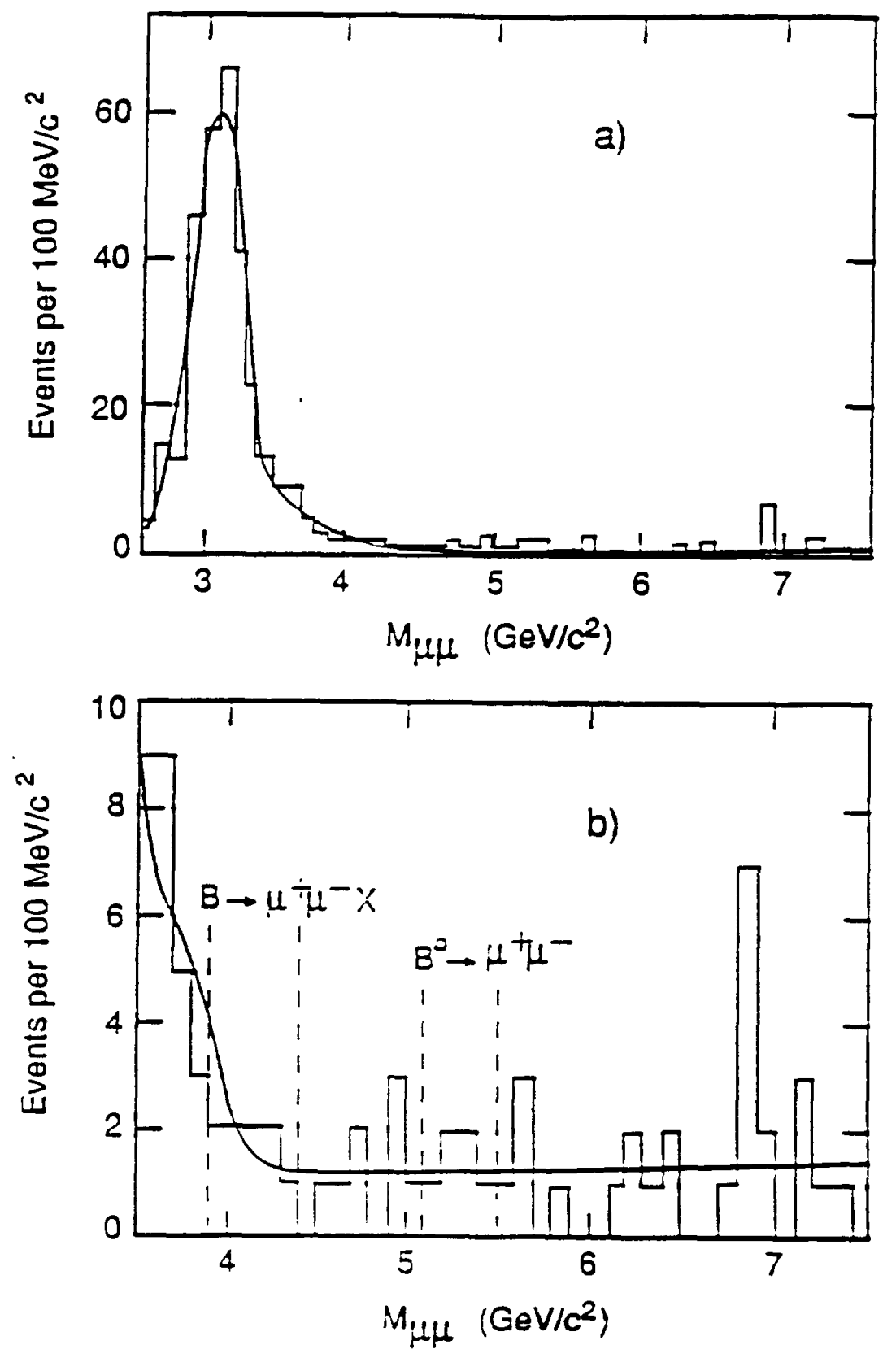

Fig. 4: The mass distribution for oppositely charged tracks associated to a dimuon with $3.9 \mathrm{GeV} / \mathrm{c}^{2}<$ $7 \mathrm{GeV} / \mathrm{c}$ : (a) the region over which the phenomenological fit was performed, (b) an expanded view showing the $B^{0} \rightarrow \mu^{+} \mu^{-}$and $B \rightarrow \mu^{+} \mu^{-} X$ search regions. The solid line represents the results of the phenomenological fit to the data. 


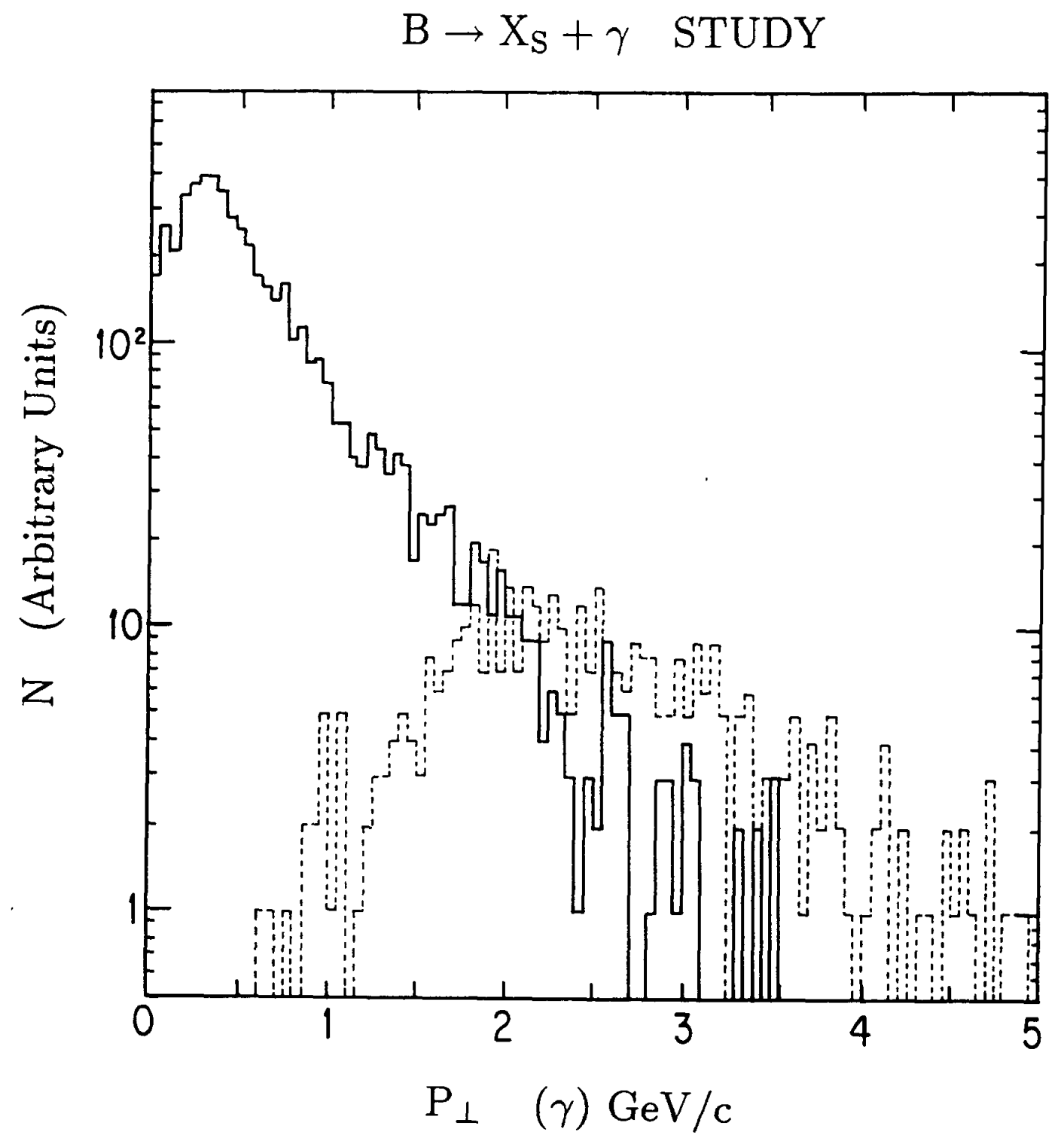

Fig. 5: The $p_{t}$ distributions of $\gamma$ 's from $250,000 B$ background events superimposed with $1,000 B$ signal events after the $\phi^{0}$ mass range cut of $0 \leq m_{\gamma \gamma} \leq 0.2 \mathrm{GeV}$ is applied with $\eta \rightarrow \gamma \gamma$ suppression. For the region $p_{t} \geq 3.6 \mathrm{GeV}$ the background is completely suppressed but the signal is statistically significant. (From the UCLA Monte Carlo study by D. Cline and J. Park, et al.) 


\section{FCNC \\ and \\ New Elementary Particles}

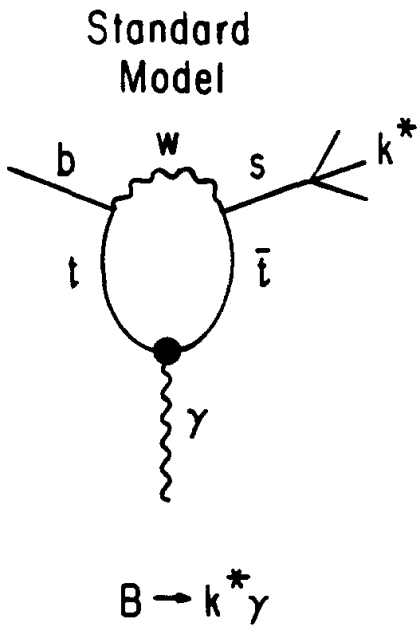

(Cornell)

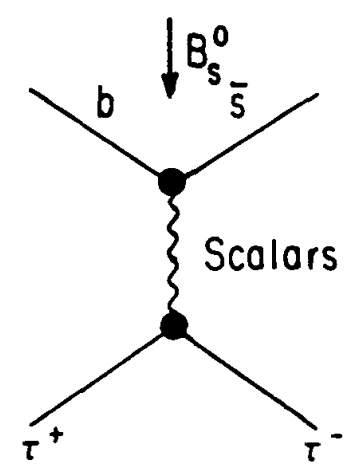

$B_{S}^{0} \rightarrow \tau^{+} \tau^{-}$
Charged

Higgs
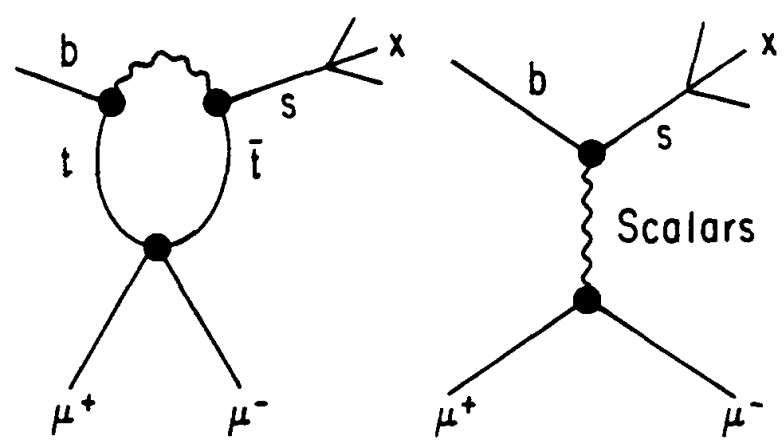

$$
B \rightarrow \mu^{+} \mu^{-} x
$$

(UAI)

$B \rightarrow \mu^{+} \mu^{-} x$

(UA1)
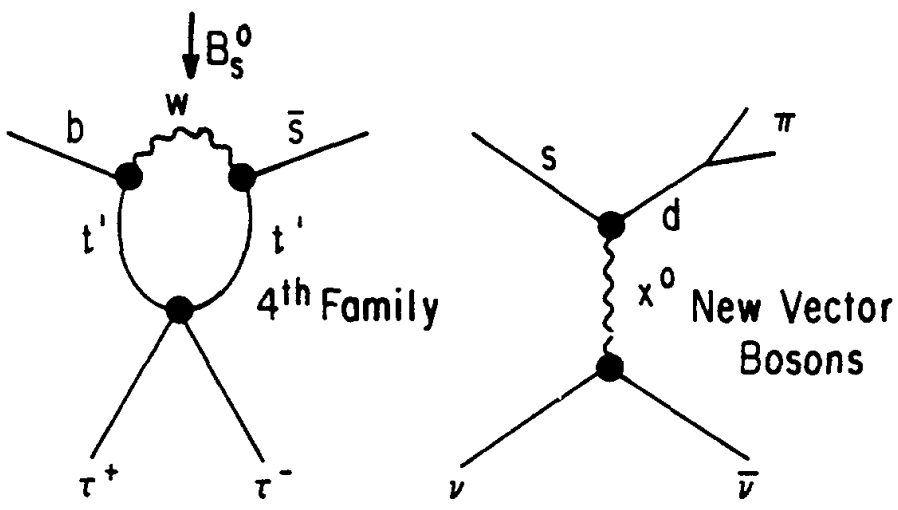

$k^{+} \rightarrow \pi^{+} \nu \bar{\nu}$

$3^{\text {rd }}$ Family

Transitions

Fig. 6: Various FCNC processes that could arise from theories "Beyond the Standard Model." 


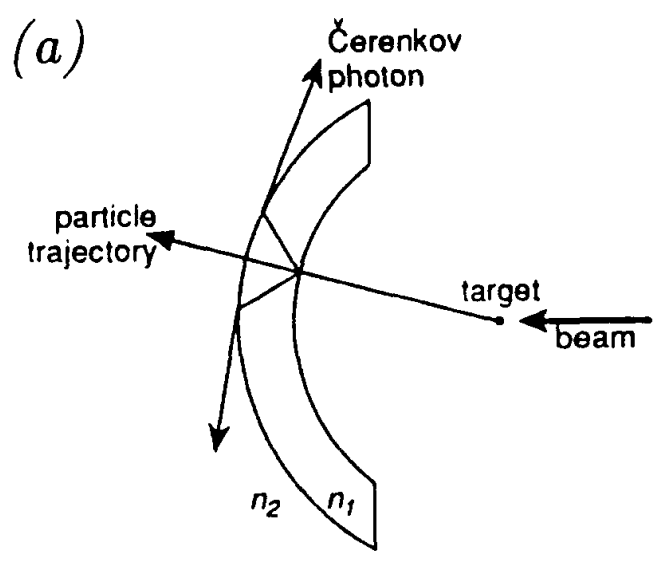

a)

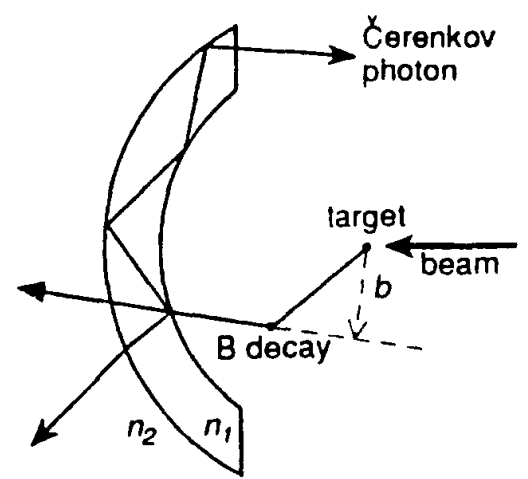

b)

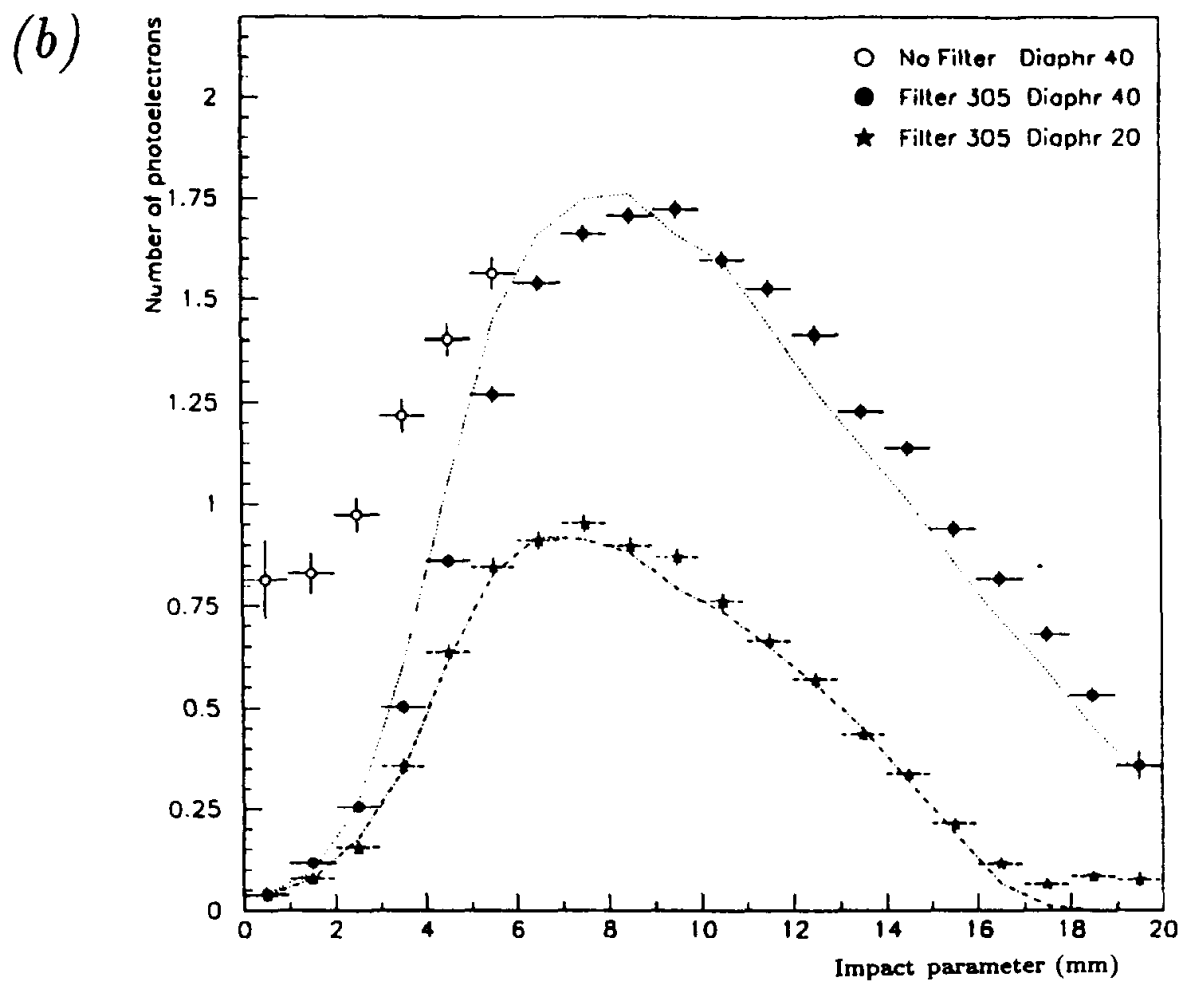

Fig. 7: (a) The principle of the optical discriminator: a particle with zero impact parameter, a), produces Cherenkov light refracted through the outer surface of the crystal, whereas, for a particle with a non-zero impact parameter, b), part of the Cherenkov light is internally reflected and trapped. (b) Amplitude of the signal as a fur.ition of the impact parameter with and without filter and with two different diagrams. 